\title{
Comparing remote atomic clocks via VLBI networks and fiber optic links: the LIFT/MetGeSp perspective
}

\author{
R. Ricci*, M. Negusini, F. Perini, C. Bortolotti, M. Roma, R. Ambrosini, G. \\ Maccaferri, M. Stagni M. Nanni, E. Kravchenko \\ INAF-Istituto di Radioastronomia \\ Bologna, Italy \\ E-mail: r.ricci@ira.inaf.itm.negusini@ira.inaf.it \\ f.perini@ira.inaf.it c.bortolotti@ira.inaf.itm.roma@ira.inaf.it \\ r.ambrosini@ira.inaf.it mstagni@ira.inaf.it m.nanni@ira.inaf.it \\ e.kravchenko@ira.inaf.it \\ D. Calonico, C Clivati, A. Tampellini, F. Levi, A. Mura \\ Istituto Nazionale di Ricerche Metrologiche (INRiM) \\ Torino, Italy \\ E-mail: d.calonicoeinrim.it c.clivatieinrim.it a.tampellinieinrim.it \\ f.levi@inrim.it a.mura@inrim.it
}

\section{G. Bianco, M. Siciliani de Cumis, L. Santamaria Amato}

ASI-Centre for Space Geodesy

Matera, Italy

E-mail: giuseppe.bianco@asi.it mario.sicilianidecumiseasi.it

luigi.santamariaeasi.it

\begin{abstract}
Very Long Baseline Interferometry experiments require an extremely precise synchronization between the atomic clocks keeping the time and frequency standards at radiotelescope observatories. Recently the availability of fiber optic links from a few radio observatories and their national metrological institutes has made possible the streaming of extremely stable frequency standards via optical atomic clocks (even two order of magnitudes better than Rubidium or Hydrogen maser standards). Firstly, I will present the infrastructure of the Italian Link for Frequency and Time (LIFT) and results of the MetGesp project aimed at finally creating a common clock between two of the antennas of the VLBI Italian Network. Secondly, I will show the results of VLBI experiments in which the phase rms noise was used to accurately compare the synchronicity of atomic clocks located at a few EVN sites (Medicina, Noto, Yebes, Torun). VLBI clock timing proves a valid alternative to satellite-based techniques such as Global Navigation Satellite System or Two-Way Satellite Frequency and Time Transfer.
\end{abstract}

14th European VLBI Network Symposium \& Users Meeting (EVN 2018)

8-11 October 2018

Granada, Spain

${ }^{*}$ Speaker. 


\section{Introduction}

Atomic clock timing can be effectively used for Very Long Baseline Interferometry (VLBI) applications, but it can also be utilized for relativistic geodesy ([1], [2]) when comparing optical clocks and in the future networks of optical clocks it will provide a redefinition of the SI second [3]. Clocks based on Cesium fountains are at the moment the primary standard in time metrology, having a stability of the order of $10^{-16}$ after a few hundred seconds of integration, but in the near future Strontium or Ytterbium lattice optical clocks will achieve a factor-of-two improvement in stability and accuracy on a such time integration. The frequency signal dissemination via optical fiber links is for the time being the most effective and stable way of comparing high performance atomic clocks on continental baselines. For intercontinental distances two techniques are being used to synchronize clocks and spread frequency standards: the Global Navigation Satellite System (GNSS) and the better performing but more expensive Two-Way Satellite Time and Frequency Transfer (TWSTFT). VLBI clock timing techniques (in particular geodetic VLBI) are now becoming a valid alternative the former two.

In mm-VLBI applications an improved frequency stability could help mitigate the loss of phase coherence between stations $\left(\delta v / v<1 / \tau \nu \leq 10^{-13}\right.$ where $v$ and $\tau$ are the observing frequency and integration time). The forthcoming Square Kilometer Array will also benefit from better synchronization techniques using fiber optics [4]. Finally a better clock spectral purity obtained from fiber-distributed frequency standards provides less Local Oscillator phase noise in both single-dish and VLBI observations: the LO phase noise scales as $\mathrm{N}^{2}$ where $\mathrm{N}$ is the number of multiplication steps from the Radio Frequency (RF) to the sky frequency (see [5]).

\section{Geodetic experiments}

The LIFT (Italian Link for Frequency and Time) infrastructure spawned the MetGeSp (Metrology for Geodesy and Space) project with the aim of disseminating a highly stable and accurate frequency signal via an optical fiber link to a series of Italian facilities. The frequency generating station is located at the Turin's Italian Metrological Institute of Metrology (INRiM). The metrological lab for relativistic geodesy in Modane (under the Frejus tunnel), the Milan Tech University, the INAF-Istituto di Radioastronomia radio station where radio and geodetic observations are performed, the Italian Lab for Non-linear Spectroscopy (LENS) in Florence where the accuracy of optical clock frequencies are tested, the Telespazio Facility in the Fucino Plain where one of the main stations of the European Galileo satellite network for global navigation is located and finally the Matera Centre for Space Geodesy are the current facilities utilizing LIFT. The main radio astronomical goal of the MetGeSp project is the creation of a common clock between the Medicina 32-m radio telescope and the Matera 20-m radio telescope, but the link is also going to to provide the Medicina radio station with accurate and stable frequency for VLBI (optical) clock experiments (see Section 3 and 4).

A detailed description of the optical fiber link would be beyond the scope of this paper. In a nut shell the RF signal generated by the INRiM clock is up-converted to the frequency of a $1.5 \mu \mathrm{m}$ laser via an opto-electronic device (an optical frequency comb) and the phase is kept synchronized via a phase-locked loop. The laser signal is beamed along a 550-km dedicated fiber. To prevent 
the signal attenuation nine sub-stations equipped with Erbium-doped Field Amplifiers (EDFAs) are set along the link. A remote control in Turin is used to minimize gain instabilities over time. A frequency stability of the order of $10^{-19}$ in terms of Allan deviation over 1000 seconds can be obtained through a round-trip servo mechanism for noise cancellation. In Medicina the laser signal is regenerated and down-converted via another optical frequency comb to the RF domain. The resulting RF (5 MHz, $10 \mathrm{MHz}$ and 1PPS) are used directly in the VLBI receiver chain and also for remote clock comparison. More details are found in [6] and [7].

The first VLBI test making use of the LIFT optical fiber link between the Medicina radio station and Turin was the geodetic experiment EUR137 in September 2015 (published in [8]): the local and remote H-maser frequency signals were alternately injected into the VLBI data acquisition chain and the data from the two clocks were analyzed as two separate experiments.

A link monitoring campaign lasting from May until early July 2017 was carried out in order to evaluate its reliability as a few unlocks were present during the EUR experiment. The link performance was good with $97 \%$ up-time. H-maser comparison between Medicina and Turin showed a stability of $\sigma_{\mathrm{y}} \simeq 2 \times 10^{-14}$ on the phase difference after $10^{3}$ seconds in terms of Allan deviation, in agreement with expectations.

In order to further test the VLBI and link set-up in view of a common-clock experiment between Medicina and Matera steations two 24 hour-long S/X-band geodetic experiments were carried out on in July 2017 and April 2018 utilizing the Medicina, Noto and Matera stations. Medicina received the frequency signal from Turin for the all duration of the runs. The data were correlated in Bologna with the local DiFX correlator [9] and fringe fitted using the Haystack Observatory Post-processing Software fourfit [10]. The geodetic tools CALC/SOLVE and nuSolve [11] were used to analyze the correlated data. In the former of two test sessions a few unlocks were found and fixed at the geodetic analysis stage. The latter geodetic session ran smoothly without any link unlocks, but problems with correlating Noto scans resulted in the usage of only the Medicina-Matera observation pairs in the geodetic analysis. The group delay weighted rms residuals after station, clock and atmosphere model subtraction are $56 \mathrm{ps}$ on the Mc-Nt baseline and $46 \mathrm{ps}$ on the full July 2017 experiment. The related plot is in Fig. 1.

\section{VLBI clock timing experiments}

An alternative way to study remote clock synchronicity using VLBI antennas involves the statistics of the interferometric phase [13]. To this end a series of VLBI clock timing experiments were performed with a network comprising the stations of Medicina, Noto, Matera, Yebes and Metsahovi in January/February 2018 (Effelsberg station accepted to take part in one run but it was wind stowed for the full time). Many factors contribute to the deterioration of the interferometric phase stability, the most important being atmospheric instabilities, gain elevation effects and antenna thermal deformations. In order to minimize these performance degrating effects the VLBI runs were performed at night during the winter months on a point-like radio source (15-min scans in 3-hour runs at medium/high antenna elevation to minimize air mass absorption). In Table 1 we report a summary of the VLBI clock timing observations.

The S/X-band observations (VT001) were performed with the stardard geodetic frequency set-up and bit rate. The C-band observations were performed with a radio astronomical VLBI 
Table 1: Summary of the VLBI clock timing observations: project codes, the observing dates, the stations involved, the bands used and whether the Medicina station was receiving or not the remote frequency standard from INRiM. Baselines with Metsahovi could not be correlated because of a data format problem.

\begin{tabular}{|l|c|l|c|c|}
\hline Project code & Date & stations & Band & Mc rem clock? \\
\hline VT001 & 20180118 & Mc,Nt,Ma,Ys,Mh & S/X & No \\
VT003 & 20180124 & Mc,Nt,Tr & C & No \\
VT005 & 20180219 & Mc,Nt,Tr & C & No \\
VT006 & 20180220 & Mc,Nt,Tr,Ys & C & Yes \\
\hline
\end{tabular}

frequency set-up: the observing band was split into 4 contiguous 8-MHz wide sub-bands (IFs) of 32 frequency channels each just below the sky frequency of $5 \mathrm{GHz}$. The Bologna DiFX correlator was used to correlate the station data which were then read into FITS files. The fringe fitting and frequency averaging were done in AIPS [14]. The data were read out from AIPS into ASCII tables and the statistics on the phase stability were computed scan-by-scan according to [13]: the scan samples were separated into couples (even statistics) and triplets (odd statistics) and then first differences and interpolated-value differences were computed together with their root mean square. To match the scheme in [13] only Right-hand polarization data were taken and analyzed. The Cband experiments had 2-bit sampling and 1-sec time integration. The central $80 \%$ of the bandpass was used in the analysis, thus removing the less sensitive sloping wings. The time synchronization was computed using the formula:

$$
\Delta t_{\mathrm{rms}}=\frac{\Delta \phi_{\mathrm{rms}}}{2 \pi v_{0}}
$$

where $\Delta t_{\mathrm{rms}}$ is the rms time synchronization between clocks, $\Delta \phi_{\mathrm{rms}}$ is the phase rms noise and $v_{0}$ is the sky centre frequency in each sub-band. The results are shown in Fig. 2. The results are in good agreement with [13] on the same timescale. We also found similar statistical values for $\Delta t_{\mathrm{rms}}$ for remote and local clocks for the Medicina station (compare VT005 and VT006 columns).

\section{Conclusions and future developments}

LIFT is an infrastructure developed to stream frequency signals from the Italian Metrological Institute (INRiM) to remote facilities via an ultra-stable optical fiber link.

Geodetic VLBI experiments are being performed with the remote frequency signal provided to the Medicina radio station by Turin's INRiM with ten's of ps in group delay wrms residuals after standard geodetic data modeling. This results are in agreement with experiments utilizing local clocks.

Rms statistics on the VLBI interferometric phase in geo/radio VLBI test experiments were successfully used to time remote and local clocks following the same scheme by [13].

The Matera radio station has just been connected to the now 1800-km long LIFT link, thus becoming the farthest end in the frequency signal dissemination infrastructure. Tests on the link stability and up-time performances are underway. A VLBI experiment involving the Mc and Ma stations operating in common-clock mode (both receiving the same frequency signal from Turin's INRiM) is expected to take place in early 2019. 


\section{Acknowledgements}

The Authors thank the telescope personnel in Medicina (A. Mattana), Matera (G. Colucci, M. Paradiso, F. Schiavone), Noto (P. Cassaro, F. Schiavone), Yebes (B. Tercero, J. Gonzalez, P. de Vicente), Torun (A. Marecki, P. Wolak, M. Gavronski), Effelsberg (A. Kraus, U. Bach) and Metsahovi (J. Kallunki, J. Tammi) for their support during the VLBI experiments.

\section{References}

[1] C. Lisdat, G. Grosche, N. Quintin, C. Shi, S.M.F. Raupach et al. A clock network for geodesy and fundamental science, Nature Communications, 7, 1-7, doi: 10.1038/ncomms12443, 2016

[2] J. Grotti, S. Koller, S. Vogt, S. Haefner, U. Sterr, et al. Geodesy and metrology with a transportable optical clock, Nature Physics, 14, 437-441, doi: 10.1038/s41567-017-0042-3, 2018

[3] F. Riehle, Optical clock networks, Nature Photonics, 11, 25-31, doi: 10.1038/nphoton.2016.235, 2017

[4] Y. He, K.G.H. Baldwin, B.J. Orr, R.B. Warrington, M.J. Wouters et al. Long-distance telecom-fibre transfer of a radio-frequency reference for radio astronomy, Optica, $\mathbf{5}, 138$, doi: 10.1364/OPTICA.5.000138, 2018

[5] M. Rioja, R. Dodson, Y. Asaki, J. Harnett, S. Tingay. The impact of Frequency Standards on Coherence in VLBI at the Highest Frequencies, Astronomical Journal, 144, 121, doi:10.1088/0004-6256/144/121, 2012

[6] D. Calonico, E.K. Bertacco, C.E. Calosso, C. Clivati, G.A. Costanzo et al. High-accuracy coherent optical frequency transfer over a doubled 642-km fibre link, Appl. Phys. B, 117, 979-986, 2014

[7] C. Clivati, G.A. Costanzo, M. Frittelli, F. Levi, A. Mura, et al. A coherent fibre-optic link for Very Long Baseline Interferometry, IEEE Trans on Ultrason. Ferroel. Freq. Contr., 62, 1907-1912, 2015

[8] C. Clivati, R. Ambrosini, T. Artz, A. Bertarini, C. Bortolotti, et al. A VLBI experiment using a remote atomic clock via a coherent fibre link, Nature Scientific Reports, 7:40992, doi:10.1038/srep40992, 2017

[9] A.T. Deller, S.J. Tingay, M. Bailes, C. West, DiFX: A Software Correlator for VLBI Using Multiprocessor Computing Environments, Publications of the Astronomical Society of the Pacific, 119, 318-336, doi: 10.1086/513572, 2007

[10] R. Cappallo, HOPS fourfit userâĂŹs manual Version 1.0, HOPS web page: https://www.haystack.mit.edu/tech/vlbi/hops.html

[11] S. Bolotin, K. Baver, J. Gipson, D. Gordon, D. MacMillan. The VLBI Data Analysis Software vSolve: Development Progress and Plans for the Future, In proceedings of the International VLBI Service for Geodesy and Astrometry 2014 General Meeting, Science Press (Beijing), pages 253-257, ISBN 978-7-03-042974-2, 2014

[12] R. Dach, S. Lutz, P. Walser, P. Fridez editors. Bernese GNSS Software Version 5.2. User manual, Astronomical Institute, University of Bern, Bern Open Publishing. DOI: 10.7892/boris.72297; ISBN: 978-3-906813-05-9, 2015

[13] P. Krehlik, L. Buczek, J. Kolodziej, M. Lipiński, L. Sliwczynski et al. Fibre-optic delivery of time and frequency to VLBI station, Astronomy \& Astrophysics, 603, 48, doi:10.1051/0004-6361/201730615, 2017 [arXiv:1703.09479]

[14] E. Greisen and A. Heck, editors, AIPS, the VLA, and the VLBA, in Information Handling in Astronomy - Historical Vistas: p. 114, 2003 AIPS web page: http://http://www.aips.nrao.edu/index.shtml 


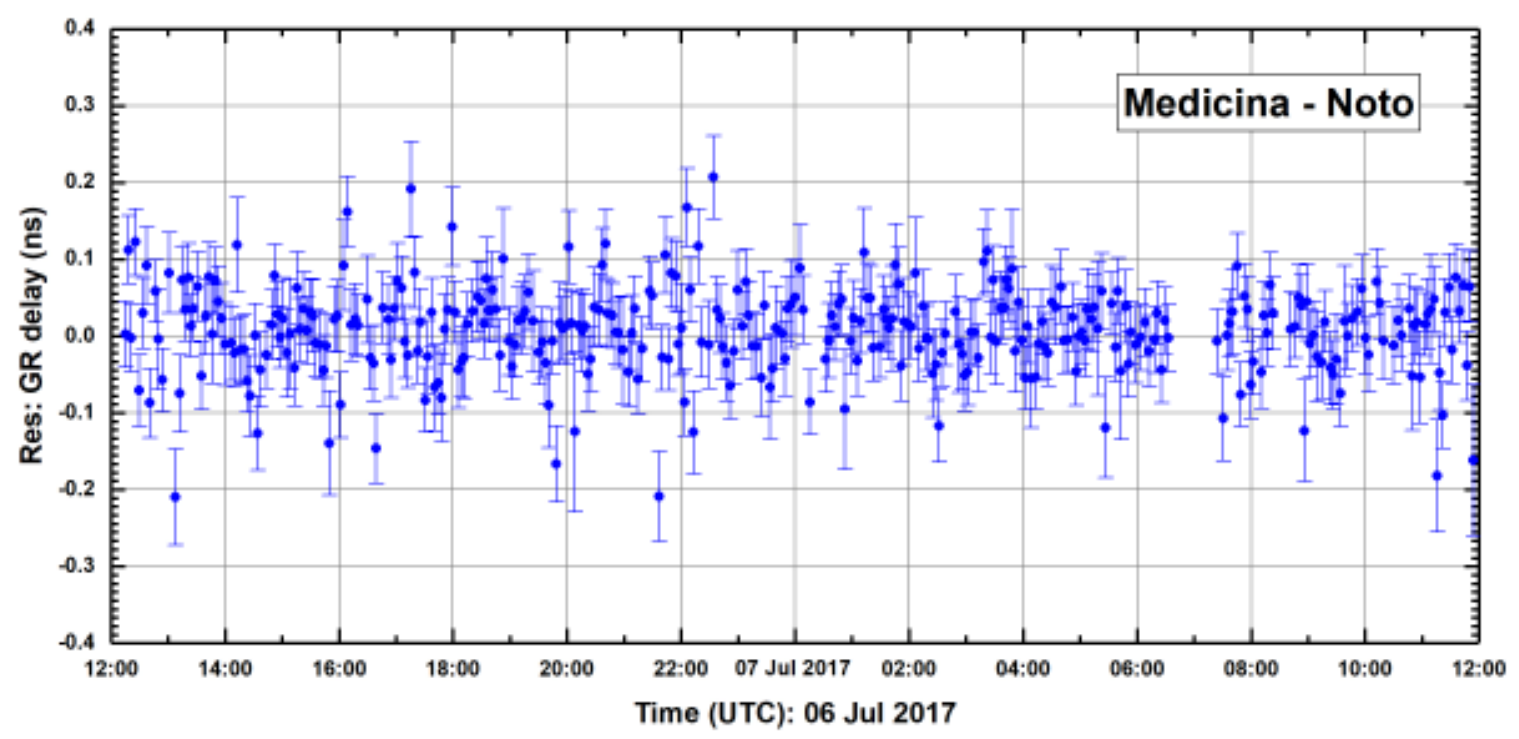

Figure 1: Group delay residuals vs observing time for July 2017 experiment.

\begin{tabular}{|c|c|c|c|c|c|c|}
\hline & & $\begin{array}{l}\text { VT005 } 19 / 02 \\
\text { scan: NoO002 }\end{array}$ & $\begin{array}{l}\text { VT006 } \\
\text { scan: }\end{array}$ & $\begin{array}{l}9 / 02 \\
\text { NoOOO2 }\end{array}$ & $\begin{array}{l}\text { VTO0 } \\
\text { scan }\end{array}$ & $\begin{array}{l}24 / 01 \\
\text { NoOOO2 }\end{array}$ \\
\hline & & Local H-maser & remote & H-maser & local $\mathrm{H}$ & naser \\
\hline & & even $\quad$ odd & even & odd & even & odd \\
\hline Mc-Nt & 1RR & $2.47(12) 2.53(15)$ & $2.74(13)$ & $2.78(16)$ & $1.25(6)$ & $1.13(7)$ \\
\hline & 2RR & $2.41(11) 2.49(14)$ & $2.65(13)$ & $2.73(16)$ & $1.74(9)$ & $1.16(7)$ \\
\hline & 3RR & $2.44(12) 2.62(15)$ & $2.66(13)$ & $2.64(15)$ & $1.36(7)$ & $1.13(7)$ \\
\hline & 4RR & $2.50(12) 2.68(16)$ & $2.63(12)$ & $2.79(16)$ & $1.35(7)$ & $1.17(7)$ \\
\hline Mc-Tr & 1RR & $1.84(11) 1.78(14)$ & $2.04(13)$ & $1.76(13)$ & $\begin{array}{l}{[450]} \\
1.26(6)\end{array}$ & $1.04(6)$ \\
\hline & $2 \mathrm{RR}$ & $1.82(11) \quad 1.75(13)$ & $2.68(17)$ & 1.99 (15) & - & - \\
\hline & 3RR & $1.84(11) 1.70(13)$ & $1.80(11)$ & $1.65(13)$ & - - & $\cdots$ \\
\hline & 4RR & $2.02(13) 1.88(14)$ & $1.83(11)$ & $1.68(13)$ & -- & -. \\
\hline & & [172] & [258] & & [450] & [300] \\
\hline $\mathrm{Nt}-\mathrm{Tr}$ & 1RR & $1.37(10)$ & $2.64(16)$ & $2.54(19)$ & $1.51(8)$ & $1.51(9)$ \\
\hline & $2 \mathrm{RR}$ & $1.58(10)$ & $3.25(20)$ & $2.86(22)$ & - & -. \\
\hline & 3RR & $1.51(9) \quad 1.52(12)$ & $2.42(15)$ & $2.24(17)$ & $\cdots$ & $\cdots$ \\
\hline & 4RR & $1.54(10) 1.58(12)$ & $2.35(15)$ & $2.21(17)$ & - & - \\
\hline & & [258] [172] & [258] & [172] & [450] & [300] \\
\hline
\end{tabular}

Figure 2: The $\Delta t_{\mathrm{rms}}$ in ps from the phase rms stats in the VT003, VT005 and VT006 experiments. Statistical errors are in round brackets. The sample numbers on which the stats are computed are in square brackets. 